\title{
Distributed State Estimation for Uncertain Markov-type Sensor Networks with Mode-dependent Distributed Delays
}

\author{
Jinling Liang*, Zidong Wang and Xiaohui Liu
}

\begin{abstract}
In this paper, the distributed state estimation problem is investigated for a class of sensor networks described by uncertain discrete-time dynamical systems with Markovian jumping parameters and distributed time-delays. The sensor network consists of sensor nodes characterized by a directed graph with a nonnegative adjacency matrix that specifies the interconnection topology (or the distribution in the space) of the network. Both the parameters of the target plant and the sensor measurements are subject to the switches from one mode to another at different times according to a Markov chain. The parameter uncertainties are norm-bounded that enter into both the plant system as well as the network outputs. Furthermore, the distributed time-delays are considered which are also dependent on the Markovian jumping mode. Through the measurements from a small fraction of the sensors, this paper aims to design state estimators that allow the nodes of the sensor network to track the states of the plant in a distributed way. It is verified that such state estimators do exist if a set of matrix inequalities are solvable. A numerical example is provided to demonstrate the effectiveness of the designed distributed state estimators.
\end{abstract}

\section{Keywords}

Distributed state estimation, sensor network, parameter uncertainties, Markovian chain, mode-dependent distributed delays.

\section{INTRODUCTION}

In the past few years, theoretical research and practical applications of sensor networks have received increasing research attention from a variety of areas including military sensing, physical security, air traffic control, distributed robotics, as well as industrial and manufacturing automation. Roughly speaking, a sensor network consists of a number of sensor nodes distributed over a spatial region. Each sensor node has wireless communication capability, some level of intelligence for signal processing and for disseminating data. These sensor nodes collaborate amongst themselves to set up a sensing network [3,27]. A typical problem in sensor networks, known as the distributed estimation problem, is how to estimate the state of the dynamic process (plant target) from spatially distributed nodes that form a wireless ad hoc network with every node having its own notion of time. In other words, for distributed estimation problems, the individual sensor in a sensor network locally estimates the system state from not only its own measurement but also its neighboring sensors'

This work was supported in part by the Royal Society of the U.K., the National Natural Science Foundation of China under Grants 60804028 and 61028008, the Specialized Research Fund for the Doctoral Program of Higher Education for New Teachers in China under Grant 200802861044, the Teaching and Research Fund for Excellent Young Teachers at Southeast University of China, the International Science and Technology Cooperation Project of China under Grant No. 2009DFA32050, and the Alexander von Humboldt Foundation of Germany.

J. Liang is with the Department of Mathematics, Southeast University, Nanjing 210096, China.

Z. Wang and X. Liu are with the Department of Information Systems and Computing, Brunel University, Uxbridge, Middlesex, UB8 3PH, United Kingdom. (Email: Zidong.Wang@brunel.ac.uk)

${ }^{*}$ Corresponding author. (Email: jinlliang@gmail.com) 
measurements according to the given topology. Fundamentally different from the traditional central filtering techniques $[9,12,21,24,26,29]$, the difficulty in designing distributed estimation algorithms stems from 1) the complicated coupling between the sensor nodes according to a given topology; and 2) the network-induced phenomena such as distributed communication delays and Markov-type mode switches.

Owing to its importance in collaborative signal processing to efficiently process distributed information gathered, the distributed estimation problem has gained particular research interests in recent years. For example, in $[18,19]$, a distributed Kalman filtering algorithm has been developed with different sensing models and different consensus strategies. In [22], a distributed estimation algorithm has been proposed to adaptively update the weights for minimizing the estimation error variance. An alternative multiscale approach has been put forward in [11] to accelerate the convergence of the decentralized consensus problem for networks with poor connectivity. In [28], a new type of distributed consensus filter is designed, where each sensor can communicate with the neighboring sensors and filtering can be performed in a distributed way. More recently, in $[5,6]$, the distributed diffusion filtering algorithm has been designed with the information diffused across the network through a sequence of Kalman iterations and data-aggregation. Despite the popularity of the investigation on various distributed estimation algorithms, the network-induced phenomena that are directly associated with sensor networks have been largely overlooked due probably to the mathematical complexity, which motivates our present research.

For wireless sensor networks, the network size, the communication constraints as well as the stringent energy limit give rise to great challenges to classical estimation techniques that demand enormous storage space and centralized computation. As such, it is not surprising that distributed estimation algorithms have recently stirred considerable research attention. Nevertheless, there remain some open issues outlined as follows that deserve further investigation. 1) A sensor network is often subject to the mode switch according to a Markov chain. In [8], a sensor network has been shown to have jumping behavior due to the network's working environment (normal or hazardous) and the mobility of the sensor node. In [10], it has been concluded that the shuffle-exchange networks can model practical interconnection systems due to their size of switching (jumping) elements and uncomplicated configuration. 2) The distributed delays occur naturally in sensor networks since the wireless information transmission has a spatial nature due to the presence of an amount of parallel pathways of a variety of node sizes and lengths. Note that, for sensor networks, the distributed delay typically takes place in a digital (discrete) way, see [16] for more details about the discrete distributed time-delays. 3) In practice, the actual spatial positions of sensor nodes at the experimentation stage may be uncertain to some extent and randomly fluctuate around some locations specified at the configuration stage [20], and the parameters of the modeled sensor networks are inevitably subject to uncertainties. Unfortunately, up to now, the unavoidable issues of mode switching, distributed delays as well as parameter uncertainties have not yet been investigated for the distributed state estimation problem of sensor networks. It is, therefore, the main aim of this paper to shorten such a gap by launching an initial study on the distributed state estimation for uncertain Markov-type sensor networks with mode-dependent distributed delays.

In this paper, attention is focused on the distributed state estimation problem for a class of sensor networks described by uncertain discrete-time stochastic systems with Markovian jumping parameters and modedependent distributed delays. Through available output measurements from each individual sensor, we wish to design distributed state estimators to approximate the states of the networked dynamic system based on the sensor's and its neighboring sensors' measurements according to certain topology. Sufficient conditions are presented to guarantee the convergence of the estimation error systems for all admissible parameter un- 
certainties and distributed delays for each mode. The explicit expression of individual estimator is derived to facilitate the distributed computing of state estimation from each sensor. Finally, numerical example is given to verify the theoretical results.

The remaining part of the paper is organized as follows. In Section II, the distributed sate estimation problem is formulated for the sensor network with Markov-type uncertain parameters and distributed delays, and some preliminaries are briefly outlined. In Section III, by resorting to the inequality techniques combining with a novel Lyapunov-Krasovskii functional constructed to reflect the mode-dependent time delays, we conduct the analysis to obtain several sufficient criteria which not only ensure the estimation error systems to be globally asymptotically stable in the mean square sense but also characterize the explicit expression of the estimator gains. In Section IV, a numerical example is given to show the applicability of the acquired conditions. Finally, conclusions are drawn in Section V.

Notations. The notation used throughout this paper is fairly standard. $\mathbb{R}^{n}$ and $\mathbb{R}^{m \times n}$ denote the $n$ dimensional Euclidean space and the set of all $m \times n$ real matrices, respectively. $\mathbb{N}$ is used to be the set $\{1,2, \ldots\}$. $I$ and 0 represent, respectively, the identity matrix and the zero matrix of appropriate dimensions. The Kronecker product of matrices $A \in \mathbb{R}^{m \times n}$ and $B \in \mathbb{R}^{p \times q}$ is a matrix in $\mathbb{R}^{m p \times n q}$ and denoted as $A \otimes B$. The notation $X>0$ for $X \in \mathbb{R}^{n \times n}$ means that matrix $X$ is real, symmetric and positive $\operatorname{definite} \cdot \operatorname{diag}(\cdots)$ and $\operatorname{col}(\cdots)$ stand for, respectively, the block-diagonal matrix and the matrix column with blocks given by the matrices in $(\cdots) .\|\cdot\|$ refers to the Euclidean vector norm and the asterisk ' $*$ ' in a symmetric matrix is used to denote the term that is induced by symmetry. For notation $(\Omega, \mathcal{F}, \mathscr{P})$, $\Omega$ represents the sample space, $\mathcal{F}$ is the $\sigma$-algebra of subsets of the sample space and $\mathscr{P}$ is the probability measure on $\mathcal{F}$. $\mathbb{E}\left\{\alpha_{1}\right\}$, $\mathbb{E}\left\{\alpha_{1} \mid \alpha_{2}\right\}$ mean, respectively, the mathematical expectation of the stochastic variable $\alpha_{1}$ and the expectation of $\alpha_{1}$ conditional on $\alpha_{2}$. Matrices, if not stated, are assumed to be compatible for algebraic operations.

\section{Problem Formulation AND SOME PRELiminaries}

Let the complete probability space $(\Omega, \mathcal{F}, \mathscr{P})$ be fixed. Consider the Markov-type uncertain dynamic system (target plant) with $n$ modes described by the following discrete-time model:

$$
x(k+1)=D(k, r(k)) x(k)+B(k, r(k)) f(x(k))+W(k, r(k)) \sum_{v=1}^{\tau(r(k))} g(x(k-v))
$$

with $N$ sensors characterized by:

$$
y_{i}(k)=C_{i}(k, r(k)) x(k), \quad i=1,2, \ldots, N
$$

where $k \in \mathbb{N} ; x(k) \in \mathbb{R}^{n_{x}}$ is the state of the target; $y_{i}(k) \in \mathbb{R}^{n_{y}}$ is the measurement output from sensor $i$ of the target plant; $v$ is the time-delay; $\{r(k): k \in \mathbb{N}\}$ is a discrete-time homogeneous Markov chain which governs the switches among the different system modes and takes values in a finite mode set $\mathcal{S} \triangleq\{1,2, \ldots, n\}$ with mode transition probability matrix $\Pi \triangleq\left\{\pi_{l m}\right\}_{n \times n}$ given by

$$
\mathscr{P}\{r(k+1)=m \mid r(k)=l\}=\pi_{l m},
$$

where $\pi_{l m} \geq 0(l, m \in \mathcal{S})$ is the transition rate from mode $l$ to mode $m$ and, for every $l \in \mathcal{S}, \sum_{m=1}^{n} \pi_{l m}=1$. $\tau(r(k))$ characterizes the distributed time-delay dependent on the system mode $r(k) ; f(\cdot)$ and $g(\cdot)$ are nonlinear functions; $D(k, r(k)), B(k, r(k)), W(k, r(k))$ and $C_{i}(k, r(k))(i=1,2, \ldots, N)$ are time-varying matrices defined 
on the Markov chain of the form

$$
\begin{aligned}
& D(k, r(k))=D(r(k))+\Delta D(k, r(k)), \quad B(k, r(k))=B(r(k))+\Delta B(k, r(k)), \\
& W(k, r(k))=W(r(k))+\Delta W(k, r(k)), \quad C_{i}(k, r(k))=C_{i}(r(k))+\Delta C_{i}(k, r(k)),
\end{aligned}
$$

in which the constant matrices $D(r(k)), B(r(k)), W(r(k))$ and $C_{i}(r(k))$ are known, while $\Delta D(k, r(k))$, $\Delta B(k, r(k)), \Delta W(k, r(k))$ and $\Delta C_{i}(k, r(k))$ are unknown matrices representing the time-varying parameter uncertainties satisfying the following conditions:

$$
\begin{aligned}
& {[\Delta D(k, r(k)), \Delta B(k, r(k)), \Delta W(k, r(k))]=E_{1}(r(k)) F_{1}(k, r(k))\left[M_{1}(r(k)), M_{2}(r(k)), M_{3}(r(k))\right],} \\
& \Delta C_{i}(k, r(k))=E_{2 i}(r(k)) F_{2}(k, r(k)) M_{4}(r(k)) ;
\end{aligned}
$$

where $E_{1}(r(k)), E_{2 i}(r(k))$ and $M_{l}(r(k))(l=1,2,3,4)$ are known real constant matrices and $F_{1}(k, r(k))$, $F_{2}(k, r(k))$ are unknown time-varying matrix-valued functions subject to the following conditions:

$$
F_{l}^{T}(k, r(k)) F_{l}(k, r(k)) \leq I, \quad l=1,2 ; r(k) \in \mathcal{S}, k \in \mathbb{N} .
$$

Remark 1: In practice, the sensor networks often exhibit the special characteristic of Markov mode switching, that is, the sensor networks sometimes have finite modes that switch from one to another at different times, and such a switching (or jumping) can be governed by a Markovian chain. For example, the bufferless packet switching of trees and leveled networks has been illustrated in [4] to be achievable with certain network topologies. In model (1), the switching signal $\{r(k): k \in \mathbb{N}\}$ in the target plant could be obtained through statistical experiments. Note that the Markovian jumping system has been well studied for $H_{\infty}$ control and filtering problems, see e.g. [1] and [21] for discrete- and continuous-time linear systems with mode-dependent delays. For more information about the mode-dependent time-delay systems, we refer to [13-15].

In this paper, consider the sensor network consisting of $N$ sensor nodes characterized by a directed graph $\mathscr{G}=(\mathcal{V}, \mathcal{E})$ with a nonnegative adjacency matrix $\mathcal{A}=\left(a_{i j}\right)_{N \times N}$ that specifies the interconnection topology (or the distribution in the space) of the sensor network. Here, $\mathcal{V}=\{1,2, \ldots, N\}$ denotes the set of sensor nodes and $\mathcal{E} \subseteq \mathcal{V} \times \mathcal{V}$ is the set of edges. Edge $(i, j) \in \mathcal{E}$ means that there is information transmission from sensor $j$ to sensor $i$ which is represented equivalently by $a_{i j}>0$. Moreover, it is also assumed in this paper that the sensors are self-connected, i.e., $a_{i i}=1$ for all $i \in \mathcal{V}$. Node $j$ is called one of the neighbors of node $i$ if $(i, j) \in \mathcal{E}$. According to the given network topology, in the sensor network, node $i \in \mathcal{V}$ can receive information from its neighboring nodes $j \in \mathcal{N}_{i}$, where $\mathcal{N}_{i} \triangleq\{j \in \mathcal{V} \mid(i, j) \in \mathcal{E}\}$.

It should be noted that, in practice, there are a huge amount of sensors that can measure the target state $x(k)$ from measurement outputs $y_{i}(k)(i=1,2, \ldots, N)$. However, it is usually impossible to have only one centralized processor to collect the measurements from all the sensors, especially in a remote area. Therefore, it is more appropriate to use local information achieved from neighbors in a distributed way. The objective of this paper, accordingly, is to design a distributed state estimator to track the state $x(k)$ of the target plant with an acceptable accuracy.

Construct the following state estimator on sensor node $i$ :

$$
\begin{aligned}
\hat{x}_{i}(k+1)= & D(r(k)) \hat{x}_{i}(k)+B(r(k)) f\left(\hat{x}_{i}(k)\right)+W(r(k)) \sum_{v=1}^{\tau(r(k))} g\left(\hat{x}_{i}(k-v)\right) \\
& +\sum_{j \in \mathcal{N}_{i}} a_{i j} K_{i j}(r(k))\left[y_{j}(k)-C_{j}(r(k)) \hat{x}_{j}(k)\right],
\end{aligned}
$$


where $\hat{x}_{i}(k) \in \mathbb{R}^{n_{x}}$ is the estimate for the plant state $x(k)$ on the node $i$, and $K_{i j}(r(k)) \in \mathbb{R}^{n_{x} \times n_{y}}$ is the estimator gain matrix to be determined.

Letting $\tilde{x}_{i}(k)=x(k)-\hat{x}_{i}(k)$ be the state estimation error, it follows from (1), (2) and (6) that

$$
\begin{aligned}
\tilde{x}_{i}(k+1)= & D(r(k)) \tilde{x}_{i}(k)+\Delta D(k, r(k)) x(k)+B(r(k)) \tilde{f}\left(\tilde{x}_{i}(k)\right)+\Delta B(k, r(k)) f(x(k)) \\
& +W(r(k)) \sum_{v=1}^{\tau(r(k))} \tilde{g}\left(\tilde{x}_{i}(k-v)\right)+\Delta W(k, r(k)) \sum_{v=1}^{\tau(r(k))} g(x(k-v)) \\
& -\sum_{j \in \mathcal{N}_{i}} a_{i j} K_{i j}(r(k))\left[C_{j}(r(k)) \tilde{x}_{j}(k)+\Delta C_{j}(k, r(k)) x(k)\right], \quad i=1,2, \ldots, N
\end{aligned}
$$

where $\tilde{f}\left(\tilde{x}_{i}(k)\right)=f(x(k))-f\left(\hat{x}_{i}(k)\right)$ and $\tilde{g}\left(\tilde{x}_{i}(k-v)\right)=g(x(k-v))-g\left(\hat{x}_{i}(k-v)\right)$. By using the notation of Kronecker product, the estimation error dynamics governed by (7) can be rewritten in a compact form as follows:

$$
\begin{aligned}
\tilde{x}(k+1)= & \left(I_{N} \otimes D(r(k))-\bar{K}(r(k)) \tilde{C}(r(k))\right) \tilde{x}(k)+(\Delta \tilde{D}(k, r(k))-\bar{K}(r(k)) \Delta \bar{C}(k, r(k))) x(k) \\
& +\left(I_{N} \otimes B(r(k))\right) F(\tilde{x}(k))+\Delta \tilde{B}(k, r(k)) f(x(k)) \\
& +\left(I_{N} \otimes W(r(k))\right) \sum_{v=1}^{\tau(r(k))} G(\tilde{x}(k-v))+\Delta \tilde{W}(k, r(k)) \sum_{v=1}^{\tau(r(k))} g(x(k-v)),
\end{aligned}
$$

where $\tilde{x}(k)=\operatorname{col}\left(\tilde{x}_{1}(k), \tilde{x}_{2}(k), \ldots, \tilde{x}_{N}(k)\right), \tilde{C}(r(k))=\operatorname{diag}\left(C_{1}(r(k)), C_{2}(r(k)), \ldots, C_{N}(r(k))\right)$,

$$
\begin{gathered}
\Delta \tilde{D}(k, r(k))=[\underbrace{(\Delta D(k, r(k)))^{T},(\Delta D(k, r(k)))^{T}, \ldots,(\Delta D(k, r(k)))^{T}}_{N}]^{T}, \\
\Delta \tilde{B}(k, r(k))=[\underbrace{(\Delta B(k, r(k)))^{T},(\Delta B(k, r(k)))^{T}, \ldots,(\Delta B(k, r(k)))^{T}}_{N}]^{T}, \\
\Delta \tilde{W}(k, r(k))=[\underbrace{(\Delta W(k, r(k)))^{T},(\Delta W(k, r(k)))^{T}, \ldots,(\Delta W(k, r(k)))^{T}}_{N}]^{T} \\
F(\tilde{x}(k))=\left[\begin{array}{c}
\tilde{f}\left(\tilde{x}_{1}(k)\right) \\
\tilde{f}\left(\tilde{x}_{2}(k)\right) \\
\vdots \\
\tilde{f}\left(\tilde{x}_{N}(k)\right)
\end{array}\right], G(\tilde{x}(k-v))=\left[\begin{array}{c}
\tilde{g}\left(\tilde{x}_{1}(k-v)\right) \\
\tilde{g}\left(\tilde{x}_{2}(k-v)\right) \\
\vdots \\
\tilde{g}\left(\tilde{x}_{N}(k-v)\right)
\end{array}\right], \Delta \bar{C}(k, r(k))=\left[\begin{array}{c}
\Delta C_{1}(k, r(k)) \\
\Delta C_{2}(k, r(k)) \\
\vdots \\
\Delta C_{N}(k, r(k))
\end{array}\right]
\end{gathered}
$$

and $\bar{K}(r(k))=\left(a_{i j} K_{i j}(r(k))\right)_{N \times N}$ is a sparse matrix satisfying $\bar{K}(r(k)) \in \mathscr{W}_{n_{x} \times n_{y}}$, where $\mathscr{W}_{n_{x} \times n_{y}}$ is defined as

$$
\mathscr{W}_{n_{x} \times n_{y}}=\left\{\bar{U}=\left[U_{i j}\right] \in \mathbb{R}^{n_{x} N \times n_{y} N} \mid U_{i j} \in \mathbb{R}^{n_{x} \times n_{y}}, U_{i j}=0 \text { if } j \notin \mathcal{N}_{i}\right\}
$$

Throughout this paper, the following assumptions are made on the nonlinear functions $f(\cdot)$ and $g(\cdot)$ in the target system (1).

Assumption 1: $f(0)=0, g(0)=0$.

Assumption 2: There exist matrices $\Sigma_{1}$ and $\Sigma_{2}$ such that

$$
\|f(u)-f(v)\| \leq\left\|\Sigma_{1}(u-v)\right\|, \quad\|g(u)-g(v)\| \leq\left\|\Sigma_{2}(u-v)\right\| ; \quad \forall u, v \in \mathbb{R}^{n_{x}}
$$


According to Assumption 2, one can easily obtain that the nonlinear functions $F(\cdot)$ and $G(\cdot)$ defined in (8) satisfy the following conditions

$$
\|F(\tilde{x}(k))\| \leq\left\|\tilde{\Sigma}_{1} \tilde{x}(k)\right\|, \quad \| G\left(\tilde{x}(k)\|\leq\| \tilde{\Sigma}_{2} \tilde{x}(k) \| ;\right.
$$

where $\tilde{\Sigma}_{1}=\operatorname{diag}\left(\Sigma_{1}, \Sigma_{1}, \ldots, \Sigma_{1}\right)$ and $\tilde{\Sigma}_{2}=\operatorname{diag}\left(\Sigma_{2}, \Sigma_{2}, \ldots, \Sigma_{2}\right)$.

The initial condition associated with (8) is given as

$$
\tilde{x}(s)=\varphi(s) \in \mathbb{R}^{n_{x} N}, \quad s=-\bar{\tau},-\bar{\tau}+1, \ldots, 0
$$

where $\bar{\tau}=\max \{\tau(1), \tau(2), \ldots, \tau(n)\}$.

Let $\{\tilde{x}(k ; \varphi) ; k \in \mathbb{N}\}$ denote the corresponding state trajectory of the uncertain error system (8).

Definition 1: The system (6) is said to be a robustly distributed state estimator of the Markov-type dynamic system (1) with measurement outputs (2) if the estimation error system (8) is robustly asymptotically stable in the mean square sense, i.e., for any initial condition $\varphi(\cdot)$ and for all parameter uncertainties satisfying (4)-(5), the following equality

$$
\lim _{k \rightarrow \infty} \mathbb{E}\left\{\|\tilde{x}(k ; \varphi)\|^{2}\right\}=0
$$

holds for the corresponding solution $\{\tilde{x}(k ; \varphi) ; k \in \mathbb{N}\}$ of the error system (8).

Our main aim in this paper is to choose suitable estimator gain matrices $K_{i j}(r(k))$ such that the estimation error system (8) is robustly asymptotically stable in the mean square sense. Before deriving the main results, two useful lemmas are given as follows.

Lemma 1: Let $X, Y$ and $F$ be real matrices of appropriate dimensions with $F$ satisfying $F^{T} F \leq I$. Then, for any scalar $\varepsilon>0$,

$$
X F Y+(X F Y)^{T} \leq \varepsilon^{-1} X X^{T}+\varepsilon Y^{T} Y .
$$

Lemma 2: [16] Let $Q \in \mathbb{R}^{n \times n}$ be a positive semi-definite matrix, $x_{i} \in \mathbb{R}^{n}$ and scalar constant $b_{i} \geq 0(i=$ $1,2, \ldots)$. If the series concerned is convergent, then the following inequality holds:

$$
\left(\sum_{i=1}^{+\infty} b_{i} x_{i}\right)^{T} Q\left(\sum_{i=1}^{+\infty} b_{i} x_{i}\right) \leq\left(\sum_{i=1}^{+\infty} b_{i}\right) \sum_{i=1}^{+\infty} b_{i} x_{i}^{T} Q x_{i} .
$$

\section{MAIN RESUltS}

In this section, by utilizing the Lyapunov stability theory and the matrix inequality technique, sufficient conditions are to be derived which not only ensure the estimation error system (8) to be robustly asymptotically stable in the mean square sense but also characterize the explicit expression of the estimator gains.

Taking the augmented state vector as $e(k)=\operatorname{col}(x(k), \tilde{x}(k))$, the combination of (1) and (8) leads to the following augmented system:

$$
e(k+1)=\mathcal{D}(k, r(k)) e(k)+\mathcal{B}(k, r(k)) \mathscr{F}(e(k))+\mathcal{W}(k, r(k)) \sum_{v=1}^{\tau(r(k))} \mathcal{G}(e(k-v)),
$$

where $\mathcal{D}(k, r(k))=\mathcal{D}(r(k))+\Delta \mathcal{D}(k, r(k)), \mathcal{B}(k, r(k))=\mathcal{B}(r(k))+\Delta \mathcal{B}(k, r(k)), \mathcal{W}(k, r(k))=\mathcal{W}(r(k))+$ $\Delta \mathcal{W}(k, r(k))$ and

$$
\begin{aligned}
& \mathcal{D}(r(k))=\left[\begin{array}{cc}
D(r(k)) & 0 \\
0 & I_{N} \otimes D(r(k))-\bar{K}(r(k)) \tilde{C}(r(k))
\end{array}\right], \\
& \mathcal{B}(r(k))=\left[\begin{array}{cc}
B(r(k)) & 0 \\
0 & I_{N} \otimes B(r(k))
\end{array}\right], \quad \mathcal{W}(r(k))=\left[\begin{array}{cc}
W(r(k)) & 0 \\
0 & I_{N} \otimes W(r(k))
\end{array}\right],
\end{aligned}
$$




$$
\begin{aligned}
& \Delta \mathcal{D}(k, r(k))=\left[\begin{array}{cc}
\Delta D(k, r(k)) & 0 \\
-\bar{K}(r(k)) \Delta \bar{C}(k, r(k))+\Delta \tilde{D}(k, r(k)) & 0
\end{array}\right],
\end{aligned}
$$

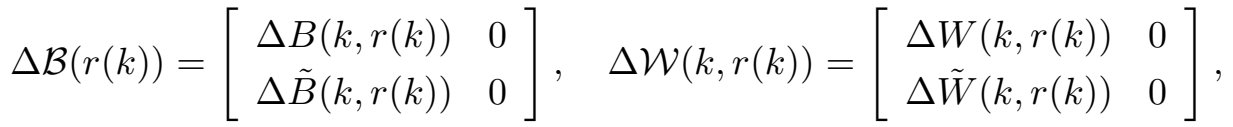

$$
\begin{aligned}
& \mathscr{F}(e(k))=\left[\begin{array}{c}
f(x(k)) \\
F(\tilde{x}(k))
\end{array}\right], \quad \mathcal{G}(e(k-v))=\left[\begin{array}{c}
g(x(k-v)) \\
G(\tilde{x}(k-v))
\end{array}\right] .
\end{aligned}
$$

In terms of Assumptions 1-2 and the properties of nonlinear functions $F(\cdot), G(\cdot)$ characterized in $(10)$, it can be easily concluded that the following inequalities

$$
\|\mathscr{F}(e(k))\| \leq\left\|\widehat{\Sigma}_{1} e(k)\right\|, \quad\|\mathcal{G}(e(k))\| \leq\left\|\widehat{\Sigma}_{2} e(k)\right\|
$$

hold, where $\widehat{\Sigma}_{1}=\operatorname{diag}\left(\Sigma_{1}, \tilde{\Sigma}_{1}\right), \widehat{\Sigma}_{2}=\operatorname{diag}\left(\Sigma_{2}, \tilde{\Sigma}_{2}\right)$ and $\tilde{\Sigma}_{1}, \tilde{\Sigma}_{2}$ are as defined in (10).

We are now ready to state the main results of this paper as follows.

Theorem 1: Under Assumptions 1-2, for the given $\bar{K}(l)(l \in \mathcal{S})$, the estimation error system (8) is robustly asymptotically stable in the mean square sense if there exist matrices $\mathcal{P}(l)>0(l \in \mathcal{S}), \mathcal{Q}>0$ and positive scalars $\varepsilon_{1}, \varepsilon_{2}, \delta_{1}, \delta_{2}$ such that the following matrix inequalities hold:

$$
\left[\begin{array}{cccc}
\Upsilon(l) & \mathcal{Z}^{T}(l) \overline{\mathcal{P}}(l) & 0 & 0 \\
* & -\overline{\mathcal{P}}(l) & \overline{\mathcal{P}}(l) \widehat{E}_{1}(l) & \overline{\mathcal{P}}(l) \widehat{E}_{2}(l) \\
* & * & -\delta_{1} I & 0 \\
* & * & * & -\delta_{2} I
\end{array}\right]<0, \quad(l \in \mathcal{S})
$$

where $\overline{\mathcal{P}}(l)=\sum_{m=1}^{n} \pi_{l m} \mathcal{P}(m), \mathcal{Z}(l)=\left[\begin{array}{llll}\mathcal{D}(l) & \mathcal{B}(l) & 0 & \mathcal{W}(l)\end{array}\right], \Upsilon(l)=\Xi(l)+\delta_{1} \aleph_{1}^{T}(l) \aleph_{1}(l)+\delta_{2} \aleph_{3}^{T}(l) \aleph_{3}(l)$,

$$
\begin{aligned}
& \widehat{E}_{1}(l)=\operatorname{col}\left(E_{1}(l), \tilde{E}_{1}(l)\right), \quad \tilde{E}_{1}(l)=[\underbrace{E_{1}^{T}(l), E_{1}^{T}(l), \ldots, E_{1}^{T}(l)}_{N}]^{T} ; \\
& \widehat{E}_{2}(l)=\operatorname{col}\left(0, \bar{K}(l) \tilde{E}_{2}(l)\right), \quad \tilde{E}_{2}(l)=[\underbrace{E_{21}^{T}(l), E_{22}^{T}(l), \ldots, E_{2 N}^{T}(l)}_{N}]^{T} \\
& \Xi(l)=\operatorname{diag}\left(-\mathcal{P}(l)+\varepsilon_{1} \widehat{\Sigma}_{1}^{T} \widehat{\Sigma}_{1}+\varepsilon_{2} \widehat{\Sigma}_{2}^{T} \widehat{\Sigma}_{2},-\varepsilon_{1} I,-\varepsilon_{2} I+(\tau(l)+\tilde{\tau}) \mathcal{Q},-\frac{1}{\tau(l)} \mathcal{Q}\right), \\
& \aleph_{1}(l)=\left[\begin{array}{llll}
\widehat{M}_{1}(l) & \widehat{M}_{2}(l) & 0 & \widehat{M}_{3}(l)
\end{array}\right], \quad \aleph_{3}(l)=\left[\begin{array}{cccc}
\widehat{M}_{4}(l) & 0 & 0 & 0
\end{array}\right], \\
& \widehat{M}_{1}(l)=\left[\begin{array}{ll}
M_{1}(l) & 0
\end{array}\right], \widehat{M}_{2}(l)=\left[\begin{array}{ll}
M_{2}(l) & 0
\end{array}\right], \widehat{M}_{3}(l)=\left[\begin{array}{ll}
M_{3}(l) & 0
\end{array}\right], \widehat{M}_{4}(l)=\left[\begin{array}{cc}
M_{4}(l) & 0
\end{array}\right]
\end{aligned}
$$

and $\tilde{\tau}=\frac{1}{2}(\bar{\tau}-\underline{\tau})(1-\underline{\pi})(\bar{\tau}+\underline{\tau}+1), \underline{\tau}=\min \{\tau(1), \tau(2), \ldots, \tau(n)\}, \underline{\pi}=\min \left\{\pi_{11}, \pi_{22}, \ldots, \pi_{n n}\right\}$.

Proof: The Schur complement lemma [2] ensures that condition (15) is equivalent to the following inequality:

$$
\left[\begin{array}{cc}
\Upsilon(l) & \mathcal{Z}^{T}(l) \overline{\mathcal{P}}(l) \\
* & -\overline{\mathcal{P}}(l)
\end{array}\right]+\delta_{1}^{-1} \aleph_{2}(l) \aleph_{2}^{T}(l)+\delta_{2}^{-1} \aleph_{4}(l) \aleph_{4}^{T}(l)<0,
$$

where $\aleph_{2}(l)=\operatorname{col}\left(0,0,0,0, \overline{\mathcal{P}}(l) \widehat{E}_{1}(l)\right)$ and $\aleph_{4}(l)=\operatorname{col}\left(0,0,0,0, \overline{\mathcal{P}}(l) \widehat{E}_{2}(l)\right)$. From the special structure of matrices $\aleph_{1}(l)$ and $\aleph_{3}(l)$, it is known that (16) is also equivalent to the following condition

$$
\left[\begin{array}{cc}
\Xi(l) & \mathcal{Z}^{T}(l) \overline{\mathcal{P}}(l) \\
* & -\overline{\mathcal{P}}(l)
\end{array}\right]+\delta_{1} \tilde{\aleph}_{1}^{T}(l) \tilde{\aleph}_{1}(l)+\delta_{2} \tilde{\aleph}_{3}^{T}(l) \tilde{\aleph}_{3}(l)+\delta_{1}^{-1} \aleph_{2}(l) \aleph_{2}^{T}(l)+\delta_{2}^{-1} \aleph_{4}(l) \aleph_{4}^{T}(l)<0,
$$


where $\tilde{\aleph}_{1}(l)=\left[\aleph_{1}(l) \quad 0\right], \tilde{\aleph}_{3}(l)=\left[\aleph_{3}(l) 0\right]$.

In order to ensure that (6) is a desired distributed state estimator of (1), we just need to show that the augmented error system (13) is robustly asymptotically stable in the mean square sense. To do so, let $\mathscr{X}(k) \triangleq\{e(k), e(k-1), \ldots, e(k-\bar{\tau})\}$ and choose the following Lyapunov-Krasovskii functional for system (13):

$$
\begin{aligned}
V(k, \mathscr{X}(k), r(k))= & V_{1}(k, \mathscr{X}(k), r(k))+V_{2}(k, \mathscr{X}(k), r(k))+V_{3}(k, \mathscr{X}(k), r(k)) \\
= & e^{T}(k) \mathcal{P}(r(k)) e(k)+\sum_{v=1}^{\tau(r(k))} \sum_{s=k-v}^{k-1} \mathcal{G}^{T}(e(s)) \mathcal{Q G}(e(s)) \\
& +(1-\underline{\pi}) \sum_{m=\underline{\tau}}^{\bar{\tau}-1} \sum_{v=1}^{m} \sum_{s=k-v}^{k-1} \mathcal{G}^{T}(e(s)) \mathcal{Q G}(e(s)),
\end{aligned}
$$

For $l \in \mathcal{S}$, we have

$$
\begin{aligned}
& \mathbb{E}\left\{V_{1}(k+1, \mathscr{X}(k+1), r(k+1)) \mid \mathscr{X}(k), r(k)=l\right\}-V_{1}(k, \mathscr{X}(k), l) \\
= & e^{T}(k+1) \overline{\mathcal{P}}(l) e(k+1)-e^{T}(k) \mathcal{P}(l) e(k) \\
= & \xi^{T}(k, l) \mathcal{Z}^{T}(k, l) \overline{\mathcal{P}}(l) \mathcal{Z}(k, l) \xi(k, l)-e^{T}(k) \mathcal{P}(l) e(k),
\end{aligned}
$$

where $\xi(k, l)=\left(e^{T}(k), \mathscr{F}^{T}(e(k)), \mathcal{G}^{T}(e(k)), \sum_{v=1}^{\tau(l)} \mathcal{G}^{T}(e(k-v))\right)^{T}$ and $\mathcal{Z}(k, l)=\left[\begin{array}{lllll}\mathcal{D}(k, l) & \mathcal{B}(k, l) & 0 & \mathcal{W}(k, l)\end{array}\right]$.

$$
\begin{aligned}
& \mathbb{E}\left\{V_{2}(k+1, \mathscr{X}(k+1), r(k+1)) \mid \mathscr{X}(k), r(k)=l\right\}-V_{2}(k, \mathscr{X}(k), l) \\
= & \sum_{m=1}^{n} \pi_{l m} \sum_{v=1}^{\tau(m)} \sum_{s=k+1-v}^{k} \mathcal{G}^{T}(e(s)) \mathcal{Q G}(e(s))-\sum_{v=1}^{\tau(l)} \sum_{s=k-v}^{k-1} \mathcal{G}^{T}(e(s)) \mathcal{Q G}(e(s)) \\
= & \sum_{m=1}^{n} \pi_{l m}\left[\sum_{v=1}^{\tau(m)} \sum_{s=k+1-v}^{k}-\sum_{v=1}^{\tau(l)} \sum_{s=k+1-v}^{k}+\sum_{v=1}^{\tau(l)} \sum_{s=k+1-v}^{k}-\sum_{v=1}^{\tau(l)} \sum_{s=k-v}^{k-1}\right] \mathcal{G}^{T}(e(s)) \mathcal{Q G}(e(s)) \\
= & \sum_{m \neq l} \pi_{l m} \sum_{v=\tau(l)+1}^{\tau(m)} \sum_{s=k+1-v}^{k} \mathcal{G}^{T}(e(s)) \mathcal{Q G}(e(s))+\sum_{v=1}^{\tau(l)}\left(\mathcal{G}^{T}(e(k)) \mathcal{Q G}(e(k))-\mathcal{G}^{T}(e(k-v)) \mathcal{Q G}(e(k-v))\right) \\
\leq & (\tau(l)+(\bar{\tau}-\underline{\tau})(1-\underline{\pi})) \mathcal{G}^{T}(e(k)) \mathcal{Q G}(e(k))-\sum_{v=1}^{\tau(l)} \mathcal{G}^{T}(e(k-v)) \mathcal{Q G}(e(k-v)) \\
& +(1-\underline{\pi}) \sum_{v=1+\underline{\tau}} \sum_{s=k+1-v}^{k-1} \mathcal{G}^{T}(e(s)) \mathcal{Q G}(e(s))
\end{aligned}
$$

and

$$
\begin{aligned}
& \mathbb{E}\left\{V_{3}(k+1, \mathscr{X}(k+1), r(k+1)) \mid \mathscr{X}(k), r(k)=l\right\}-V_{3}(k, \mathscr{X}(k), l) \\
= & (1-\underline{\pi}) \sum_{m=\underline{\tau}}^{\bar{\tau}-1} \sum_{v=1}^{m}\left(\sum_{s=k+1-v}^{k}-\sum_{s=k-v}^{k-1}\right) \mathcal{G}^{T}(e(s)) \mathcal{Q G}(e(s)) \\
= & (1-\underline{\pi}) \sum_{m=\underline{\tau}}^{\bar{\tau}-1} \sum_{v=1}^{m}\left(\mathcal{G}^{T}(e(k)) \mathcal{Q G}(e(k))-\mathcal{G}^{T}(e(k-v)) \mathcal{Q G}(e(k-v))\right) \\
= & (1-\underline{\pi})\left[\frac{1}{2}(\bar{\tau}-\underline{\tau})(\bar{\tau}-1+\underline{\tau}) \mathcal{G}^{T}(e(k)) \mathcal{Q G}(e(k))-\sum_{m=\underline{\tau}+1}^{\bar{\tau}} \sum_{s=k-m+1}^{k-1} \mathcal{G}^{T}(e(s)) \mathcal{Q G}(e(s))\right] .
\end{aligned}
$$


From Lemma 2, it is not difficult to verify that

$$
-\sum_{v=1}^{\tau(l)} \mathcal{G}^{T}(e(k-v)) \mathcal{Q G}(e(k-v)) \leq-\frac{1}{\tau(l)}\left(\sum_{v=1}^{\tau(l)} \mathcal{G}(e(k-v))\right)^{T} \mathcal{Q}\left(\sum_{v=1}^{\tau(l)} \mathcal{G}(e(k-v))\right) .
$$

At the same time, for any positive scalars $\varepsilon_{1}$ and $\varepsilon_{2}$, condition (14) ensures that the following two inequalities hold:

$$
\varepsilon_{1} e^{T}(k) \widehat{\Sigma}_{1}^{T} \widehat{\Sigma}_{1} e(k)-\varepsilon_{1} \mathscr{F}^{T}(e(k)) \mathscr{F}(e(k)) \geq 0, \quad \varepsilon_{2} e^{T}(k) \widehat{\Sigma}_{2}^{T} \widehat{\Sigma}_{2} e(k)-\varepsilon_{2} \mathcal{G}^{T}(e(k)) \mathcal{G}(e(k)) \geq 0 .
$$

With the relationships from (18) to (23), we have

$$
\begin{aligned}
& \mathbb{E}\{V(k+1, \mathscr{X}(k+1), r(k+1)) \mid \mathscr{X}(k), r(k)=l\}-V(k, \mathscr{X}(k), l) \\
\leq & \xi^{T}(k, l)\left(\Xi(l)+\mathcal{Z}^{T}(k, l) \overline{\mathcal{P}}(l) \mathcal{Z}(k, l)\right) \xi(k, l),
\end{aligned}
$$

where

$$
\mathcal{Z}(k, l)=\mathcal{Z}(l)+\Delta \mathcal{Z}(k, l) \quad \text { and } \quad \Delta \mathcal{Z}(k, l)=\left[\begin{array}{llll}
\Delta \mathcal{D}(k, l) & \Delta \mathcal{B}(k, l) & 0 & \Delta \mathcal{W}(k, l)
\end{array}\right]
$$

From the Lyapunov stability theory, one knows that if the matrix inequality $\Xi(l)+\mathcal{Z}^{T}(k, l) \overline{\mathcal{P}}(l) \mathcal{Z}(k, l)<0$ holds uniformly in $k$, we could immediately draw our conclusion. Again, from the Schur complement lemma [2], we know that

$$
\Xi(l)+\mathcal{Z}^{T}(k, l) \overline{\mathcal{P}}(l) \mathcal{Z}(k, l)<0 \Longleftrightarrow \widetilde{\Xi}(k, l) \triangleq \widetilde{\Xi}(l)+\Delta \widetilde{\Xi}(k, l)<0,
$$

where

$$
\widetilde{\Xi}(l)=\left[\begin{array}{cc}
\Xi(l) & \mathcal{Z}^{T}(l) \overline{\mathcal{P}}(l) \\
* & -\overline{\mathcal{P}}(l)
\end{array}\right], \quad \Delta \widetilde{\Xi}(k, l)=\left[\begin{array}{cc}
0 & (\Delta \mathcal{Z}(k, l))^{T} \overline{\mathcal{P}}(l) \\
* & 0
\end{array}\right] .
$$

By simple computation, it can be obtained that

$$
\begin{aligned}
\Delta \mathcal{D}(k, l) & =\left[\begin{array}{cc}
E_{1}(l) F_{1}(k, l) M_{1}(l) & 0 \\
-\bar{K}(l) \tilde{E}_{2}(l) F_{2}(k, l) M_{4}(l)+\tilde{E}_{1}(l) F_{1}(k, l) M_{1}(l) & 0
\end{array}\right] \\
& =\widehat{E}_{1}(l) F_{1}(k, l) \widehat{M}_{1}(l)-\widehat{E}_{2}(l) F_{2}(k, l) \widehat{M}_{4}(l), \\
\Delta \mathcal{B}(k, l) & =\left[\begin{array}{ll}
\Delta B(k, l) & 0 \\
\Delta \tilde{B}(k, l) & 0
\end{array}\right]=\left[\begin{array}{cc}
E_{1}(l) F_{1}(k, l) M_{2}(l) & 0 \\
\tilde{E}_{1}(l) F_{1}(k, l) M_{2}(l) & 0
\end{array}\right]=\widehat{E}_{1}(l) F_{1}(k, l) \widehat{M}_{2}(l), \\
\Delta \mathcal{W}(k, l) & =\left[\begin{array}{ll}
\Delta W(k, l) & 0 \\
\Delta \tilde{W}(k, l) & 0
\end{array}\right]=\left[\begin{array}{cc}
E_{1}(l) F_{1}(k, l) M_{3}(l) & 0 \\
\tilde{E}_{1}(l) F_{1}(k, l) M_{3}(l) & 0
\end{array}\right]=\widehat{E}_{1}(l) F_{1}(k, l) \widehat{M}_{3}(l) .
\end{aligned}
$$

Combining the above three equalities together, we can conclude that for any scalars $\delta_{1}>0$ and $\delta_{2}>0$,

$$
\begin{aligned}
\Delta \widetilde{\Xi}(k, l) & =\aleph_{2}(l) F_{1}(k, l) \tilde{\aleph}_{1}(l)+\left(\aleph_{2}(l) F_{1}(k, l) \tilde{\aleph}_{1}(l)\right)^{T}-\aleph_{4}(l) F_{2}(k, l) \tilde{\aleph}_{3}(l)-\left(\aleph_{4}(l) F_{2}(k, l) \tilde{\aleph}_{3}(l)\right)^{T} \\
& \leq \delta_{1} \tilde{\aleph}_{1}^{T}(l) \tilde{\aleph}_{1}(l)+\delta_{1}^{-1} \aleph_{2}(l) \aleph_{2}^{T}(l)+\delta_{2} \tilde{\aleph}_{3}^{T}(l) \tilde{\aleph}_{3}(l)+\delta_{2}^{-1} \aleph_{4}(l) \aleph_{4}^{T}(l),
\end{aligned}
$$

where, in the last step, Lemma 1 has been utilized to derive the inequality. Substituting (29) into the right side of the inequality in (25), we have from condition (17) that the inequality $\Xi(l)+\mathcal{Z}^{T}(k, l) \overline{\mathcal{P}}(l) \mathcal{Z}(k, l)<0$ does hold uniformly in $k$, which means that there exists a constant $\lambda<0$ such that

$$
\mathbb{E}\{V(k+1, \mathscr{X}(k+1), r(k+1)) \mid \mathscr{X}(k), r(k)=l\}-V(k, \mathscr{X}(k), l) \leq \lambda\|e(k)\|^{2} .
$$


The remaining part of the proof is similar as that in [13] and therefore omitted here. This completes the proof of the theorem.

After conducting the analysis for system (13) to be robustly asymptotically stable in the mean square sense, we are now in a position to design the estimator gains. Note that matrices $\bar{K}(l)(l \in \mathcal{S})$ contain all the information of the desired estimator parameters, to carry on, we still need the following lemma.

Lemma 3: Let $\mathbf{S}=\operatorname{diag}\left(\mathbf{S}_{\mathbf{1 1}}, \mathbf{S}_{\mathbf{2 2}}, \ldots, \mathbf{S}_{\mathbf{N N}}\right)$ with $S_{i i} \in \mathbb{R}^{n_{x} \times n_{x}}(i=1,2, \ldots, N)$ being invertible matrices. If $X=\mathbf{S} \overline{\mathbf{U}}$ for $\bar{U} \in \mathbb{R}^{n_{x} N \times n_{y} N}$, then we have $\bar{U} \in \mathscr{W}_{n_{x} \times n_{y}} \Leftrightarrow X \in \mathscr{W}_{n_{x} \times n_{y}}$.

Theorem 2: Under Assumptions 1-2, the system (6) is a robustly distributed state estimator of the Markovtype dynamic system (1) with measurement outputs (2) if there exist matrices $P_{i}(l)>0(i=1,2, \ldots, N+1 ; l \in$ $\mathcal{S}), Q_{11}>0, \mathcal{Q}_{22}>0, Q_{12}, \mathcal{X}(l) \in \mathscr{W}_{n_{x} \times n_{y}}(l \in \mathcal{S})$ and positive scalars $\varepsilon_{1}, \varepsilon_{2}, \delta_{1}, \delta_{2}$ such that the following matrix inequalities hold:

$$
\mathcal{Q} \triangleq\left[\begin{array}{cc}
Q_{11} & Q_{12} \\
* & \mathcal{Q}_{22}
\end{array}\right]>0
$$

and

$$
\Phi(l) \triangleq\left[\begin{array}{ccccccc}
\Phi_{11}(l) & \Phi_{12}(l) & 0 & \Phi_{14}(l) & \Phi_{15}(l) & 0 & 0 \\
* & \Phi_{22}(l) & 0 & \Phi_{24}(l) & \Phi_{25}(l) & 0 & 0 \\
* & * & \Phi_{33}(l) & 0 & 0 & 0 & 0 \\
* & * & * & \Phi_{44}(l) & \Phi_{45}(l) & 0 & 0 \\
* & * & * & * & \Phi_{55}(l) & \Phi_{56}(l) & \Phi_{57}(l) \\
* & * & * & * & * & -\delta_{1} I & 0 \\
* & * & * & * & * & * & -\delta_{2} I
\end{array}\right]<0, \quad(l \in \mathcal{S})
$$

where

$$
\begin{aligned}
& \Phi_{11}(l)=\operatorname{diag}\left(\Upsilon_{1}(l),-\mathcal{P}_{2}(l)+\varepsilon_{1} \tilde{\Sigma}_{1}^{T} \tilde{\Sigma}_{1}+\varepsilon_{2} \tilde{\Sigma}_{2}^{T} \tilde{\Sigma}_{2}\right), \quad \Phi_{12}(l)=\operatorname{diag}\left(\delta_{1} M_{1}^{T}(l) M_{2}(l), 0\right), \\
& \Upsilon_{1}(l)=-P_{1}(l)+\varepsilon_{1} \Sigma_{1}^{T} \Sigma_{1}+\varepsilon_{2} \Sigma_{2}^{T} \Sigma_{2}+\delta_{1} M_{1}^{T}(l) M_{1}(l)+\delta_{2} M_{4}^{T}(l) M_{4}(l), \\
& \Phi_{14}(l)=\operatorname{diag}\left(\delta_{1} M_{1}^{T}(l) M_{3}(l), 0\right), \quad \Phi_{15}(l)=\operatorname{diag}\left(D^{T}(l) \bar{P}_{1}(l),\left(I_{N} \otimes D(l)\right)^{T} \overline{\mathcal{P}}_{2}(l)-\tilde{C}^{T}(l) \mathcal{X}^{T}(l)\right), \\
& \Phi_{22}(l)=\operatorname{diag}\left(-\varepsilon_{1} I+\delta_{1} M_{2}^{T}(l) M_{2}(l),-\varepsilon_{1} I\right), \quad \Phi_{24}(l)=\operatorname{diag}\left(\delta_{1} M_{2}^{T}(l) M_{3}(l), 0\right), \\
& \Phi_{25}(l)=\operatorname{diag}\left(B^{T}(l) \bar{P}_{1}(l),\left(I_{N} \otimes B(l)\right)^{T} \overline{\mathcal{P}}_{2}(l)\right), \quad \Phi_{45}(l)=\operatorname{diag}\left(W^{T}(l) \bar{P}_{1}(l),\left(I_{N} \otimes W(l)\right)^{T} \overline{\mathcal{P}}_{2}(l)\right), \\
& \Phi_{33}(l)=\left[\begin{array}{cc}
-\varepsilon_{2} I+(\tau(l)+\tilde{\tau}) Q_{11} & (\tau(l)+\tilde{\tau}) Q_{12} \\
* & -\varepsilon_{2} I+(\tau(l)+\tilde{\tau}) \mathcal{Q}_{22}
\end{array}\right], \quad \Phi_{56}(l)=\left[\begin{array}{c}
\bar{P}_{1}(l) E_{1}(l) \\
\overline{\mathcal{P}}_{2}(l) \tilde{E}_{1}(l)
\end{array}\right], \\
& \Phi_{44}(l)=\left[\begin{array}{cc}
-\frac{1}{\tau(l)} Q_{11}+\delta_{1} M_{3}^{T}(l) M_{3}(l) & -\frac{1}{\tau(l)} Q_{12} \\
* & -\frac{1}{\tau(l)} \mathcal{Q}_{22}
\end{array}\right], \quad \Phi_{57}(l)=\left[\begin{array}{c}
0 \\
\mathcal{X}(l) \tilde{E}_{2}(l)
\end{array}\right]
\end{aligned}
$$

and $\Phi_{55}(l)=\operatorname{diag}\left(-\bar{P}_{1}(l),-\overline{\mathcal{P}}_{2}(l)\right), \mathcal{P}_{2}(l)=\operatorname{diag}\left(P_{2}(l), P_{3}(l), \ldots, P_{N+1}(l)\right), \bar{P}_{i}(l)=\sum_{m=1}^{n} \pi_{l m} P_{i}(m)(i=$ $1,2, \ldots, N+1 ; l \in \mathcal{S}), \overline{\mathcal{P}}_{2}(l)=\operatorname{diag}\left(\bar{P}_{2}(l), \bar{P}_{3}(l), \ldots, \bar{P}_{N+1}(l)\right)(l \in \mathcal{S})$, the other symbols are the same as defined in Theorem 1. Furthermore,

$$
\bar{K}(l)=\overline{\mathcal{P}}_{2}^{-1}(l) \mathcal{X}(l) ; \quad(l \in \mathcal{S})
$$

accordingly, the state estimator gains $K_{i j}(l)\left(i=1,2, \ldots, N, j \in \mathcal{N}_{i}, l \in \mathcal{S}\right)$ can be derived from (9). 
Proof: With the special structure of $\overline{\mathcal{P}}_{2}(l)$ and the restriction on matrix $\mathcal{X}(l) \in \mathscr{W}_{n_{x} \times n_{y}}$, it follows from Lemma 3 that $\overline{\mathcal{P}}_{2}^{-1}(l) \mathcal{X}(l) \in \mathscr{W}_{n_{x} \times n_{y}}$. By taking $\mathcal{P}(l)=\operatorname{diag}\left(P_{1}(l), \mathcal{P}_{2}(l)\right), \overline{\mathcal{P}}(l)=\operatorname{diag}\left(\bar{P}_{1}(l), \overline{\mathcal{P}}_{2}(l)\right)$ in Theorem 1 and noticing the condition $\overline{\mathcal{P}}_{2}(l) \bar{K}(l)=\mathcal{X}(l)$, after some computation it can be shown that

$$
\begin{aligned}
& \overline{\mathcal{P}}(l) \mathcal{D}(l)=\operatorname{diag}\left(\bar{P}_{1}(l) D(l), \overline{\mathcal{P}}_{2}(l)\left(I_{N} \otimes D(l)\right)-\mathcal{X}(l) \tilde{C}(l)\right), \\
& \overline{\mathcal{P}}(l) \mathcal{B}(l)=\operatorname{diag}\left(\bar{P}_{1}(l) B(l), \overline{\mathcal{P}}_{2}(l)\left(I_{N} \otimes B(l)\right), \quad \overline{\mathcal{P}}(l) \mathcal{W}(l)=\operatorname{diag}\left(\bar{P}_{1}(l) W(l), \overline{\mathcal{P}}_{2}(l)\left(I_{N} \otimes W(l)\right)\right),\right. \\
& \overline{\mathcal{P}}(l) \widehat{E}_{1}(l)=\left[\begin{array}{c}
\bar{P}_{1}(l) E_{1}(l) \\
\overline{\mathcal{P}}_{2}(l) \tilde{E}_{1}(l)
\end{array}\right], \quad \overline{\mathcal{P}}(l) \widehat{E}_{2}(l)=\left[\begin{array}{c}
0 \\
\mathcal{X}(l) \tilde{E}_{2}(l)
\end{array}\right] .
\end{aligned}
$$

And the corresponding result is derived easily from Theorem 1, the proof is then completed.

Remark 2: Our main results are based on the linear matrix inequality (LMI) conditions. The LMI Control Toolbox implements state-of-the-art interior-point LMI solvers. While these solvers are significantly faster than classical convex optimization algorithms, it should be kept in mind that the complexity of LMI computations remains higher than that of solving, say, a Riccati equation. For instance, problems with a thousand design variables typically take over an hour on today's workstations. However, research on LMI optimization is a very active area in the applied math, optimization and the operations research community, and substantial speed-ups can be expected in the future.

\section{Numerical Examples}

Consider a Markov-type system plant with 3 modes described by the discrete-time model (1) with

$$
\begin{aligned}
& D(1)=\left[\begin{array}{cc}
-0.3 & 0.2 \\
0.1 & -0.2
\end{array}\right], \quad D(2)=\left[\begin{array}{cc}
-0.1 & 0.2 \\
-0.1 & -0.1
\end{array}\right], \quad D(3)=\left[\begin{array}{cc}
-0.1 & 0.1 \\
0.1 & -0.1
\end{array}\right] ; \\
& B(1)=\left[\begin{array}{cc}
0.2 & 0.2 \\
0.1 & -0.2
\end{array}\right], \quad B(2)=\left[\begin{array}{cc}
0.2 & 0.2 \\
0 & 0.1
\end{array}\right], \quad B(3)=\left[\begin{array}{cc}
0.1 & 0 \\
0.2 & 0
\end{array}\right] ; \\
& W(1)=\left[\begin{array}{cc}
0.2 & -0.2 \\
0.1 & 0.2
\end{array}\right], \quad W(2)=\left[\begin{array}{cc}
0.1 & 0.2 \\
0.2 & 0
\end{array}\right], \quad W(3)=\left[\begin{array}{cc}
-0.2 & 0.1 \\
0.2 & 0.1
\end{array}\right]
\end{aligned}
$$

and $\tau(1)=3, \tau(2)=2, \tau(3)=4$; the mode transition probability matrix is given by

$$
\Pi=\left[\begin{array}{lll}
0.6 & 0.2 & 0.2 \\
0.1 & 0.8 & 0.1 \\
0.2 & 0.1 & 0.7
\end{array}\right]
$$

which means $\bar{\tau}=4, \underline{\tau}=2$ and $\underline{\pi}=0.6$. The nonlinear functions $f(\cdot)$ and $g(\cdot)$ in $(1)$ are assumed to satisfy Assumptions 1-2 with

$$
\Sigma_{1}=\left[\begin{array}{cc}
0.2 & 0.3 \\
-0.1 & 0.2
\end{array}\right], \quad \Sigma_{2}=\left[\begin{array}{cc}
0.1 & -0.2 \\
0.3 & 0.2
\end{array}\right]
$$




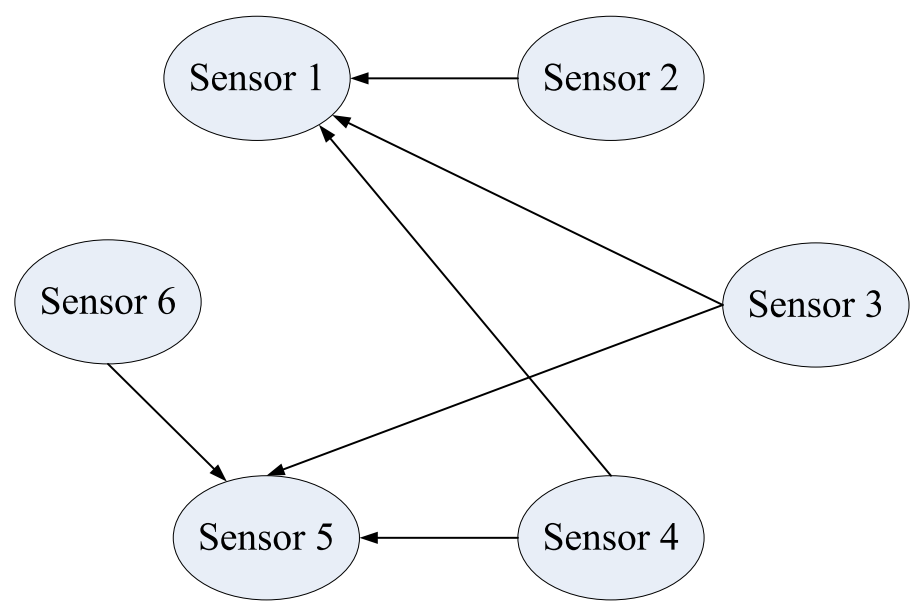

Fig. 1. Topological structure of the sensor network.

The sensor network shown in Fig. 1 consists of 6 nodes with interconnection topology characterized by the adjacency matrix

$$
\mathcal{A}=\left[\begin{array}{llllll}
1 & 1 & 1 & 1 & 0 & 0 \\
0 & 1 & 0 & 0 & 0 & 0 \\
0 & 0 & 1 & 0 & 0 & 0 \\
0 & 0 & 0 & 1 & 0 & 0 \\
0 & 0 & 1 & 1 & 1 & 1 \\
0 & 0 & 0 & 0 & 0 & 1
\end{array}\right]
$$

which illustrates that Sensor 1 receives information from Sensors 2-4, Sensor 5 receives information from Sensors 3,4,6, but Sensors 2,3,4,6 receive no information from other sensors. The dynamics of the 6 sensors are described by equation (2) with

$$
\begin{aligned}
& C_{1}(1)=\left[\begin{array}{cc}
-0.2 & 0.1 \\
-0.1 & 0.2
\end{array}\right], \quad C_{1}(2)=\left[\begin{array}{cc}
0 & 0.1 \\
0.1 & -0.1
\end{array}\right], \quad C_{1}(3)=\left[\begin{array}{cc}
0.2 & 0 \\
0 & 0.1
\end{array}\right] ; \\
& C_{2}(1)=\left[\begin{array}{cc}
0.2 & -0.2 \\
0.1 & 0.2
\end{array}\right], \quad C_{2}(2)=\left[\begin{array}{cc}
0.1 & 0.2 \\
0 & 0.1
\end{array}\right], \quad C_{2}(3)=\left[\begin{array}{cc}
0.1 & -0.1 \\
0.1 & 0.2
\end{array}\right] \text {; } \\
& C_{3}(1)=\left[\begin{array}{cc}
-0.2 & 0.1 \\
0.2 & 0.3
\end{array}\right], \quad C_{3}(2)=\left[\begin{array}{cc}
0.2 & -0.1 \\
0 & 0.1
\end{array}\right], \quad C_{3}(3)=\left[\begin{array}{cc}
-0.1 & 0 \\
0.2 & 0.1
\end{array}\right] \text {; } \\
& C_{4}(1)=\left[\begin{array}{cc}
0.2 & 0.2 \\
0 & 0.3
\end{array}\right], \quad C_{4}(2)=\left[\begin{array}{cc}
0.1 & 0 \\
-0.1 & 0.2
\end{array}\right], \quad C_{4}(3)=\left[\begin{array}{cc}
-0.1 & 0 \\
0.1 & -0.1
\end{array}\right] \text {; } \\
& C_{5}(1)=\left[\begin{array}{cc}
-0.2 & 0 \\
-0.1 & -0.1
\end{array}\right], \quad C_{5}(2)=\left[\begin{array}{cc}
-0.1 & 0.2 \\
0 & 0
\end{array}\right], \quad C_{5}(3)=\left[\begin{array}{cc}
0 & 0 \\
0.1 & -0.2
\end{array}\right] \text {; } \\
& C_{6}(1)=\left[\begin{array}{cc}
0.2 & 0.1 \\
-0.2 & 0
\end{array}\right], \quad C_{6}(2)=\left[\begin{array}{cc}
0.2 & -0.2 \\
0.1 & -0.1
\end{array}\right], \quad C_{6}(3)=\left[\begin{array}{cc}
0.1 & 0.2 \\
-0.2 & 0
\end{array}\right] \text {. }
\end{aligned}
$$


The parameter uncertainties satisfy conditions (4)-(5) with

$$
\begin{aligned}
& E_{1}(1)=\left[\begin{array}{c}
0.1 \\
-0.1
\end{array}\right], E_{1}(2)=\left[\begin{array}{l}
0.1 \\
0.2
\end{array}\right], E_{1}(3)=\left[\begin{array}{c}
0 \\
0.1
\end{array}\right] ; E_{21}(1)=\left[\begin{array}{c}
-0.1 \\
0.1
\end{array}\right], E_{21}(2)=\left[\begin{array}{c}
0.1 \\
0
\end{array}\right], \\
& E_{21}(3)=\left[\begin{array}{c}
0 \\
0.2
\end{array}\right] ; E_{22}(1)=\left[\begin{array}{c}
0 \\
0.2
\end{array}\right], E_{22}(2)=\left[\begin{array}{c}
0.1 \\
-0.1
\end{array}\right], E_{22}(3)=\left[\begin{array}{c}
-0.1 \\
0.1
\end{array}\right] ; \\
& E_{23}(1)=\left[\begin{array}{c}
0.1 \\
-0.1
\end{array}\right], E_{23}(2)=\left[\begin{array}{c}
0 \\
0.2
\end{array}\right], E_{23}(3)=\left[\begin{array}{c}
0.1 \\
0.2
\end{array}\right] ; E_{24}(1)=\left[\begin{array}{c}
0.2 \\
0.1
\end{array}\right], \\
& E_{24}(2)=\left[\begin{array}{c}
0.1 \\
0.2
\end{array}\right], E_{24}(3)=\left[\begin{array}{c}
0.1 \\
0
\end{array}\right] ; E_{25}(1)=\left[\begin{array}{c}
0.1 \\
0.2
\end{array}\right], E_{25}(2)=\left[\begin{array}{c}
-0.1 \\
0
\end{array}\right], \\
& E_{25}(3)=\left[\begin{array}{c}
0.2 \\
-0.1
\end{array}\right] ; E_{26}(1)=\left[\begin{array}{c}
0.1 \\
0
\end{array}\right], E_{26}(2)=\left[\begin{array}{c}
0.1 \\
0.2
\end{array}\right], E_{26}(3)=\left[\begin{array}{c}
0.1 \\
0.1
\end{array}\right] ; \\
& M_{1}(1)=\left[\begin{array}{ll}
0.1 & 0.2
\end{array}\right], M_{1}(2)=\left[\begin{array}{ll}
-0.1 & 0.2
\end{array}\right], M_{1}(3)=\left[\begin{array}{cc}
0 & 0.1
\end{array}\right], M_{2}(1)=\left[\begin{array}{ll}
0.2 & 0
\end{array}\right], \\
& M_{2}(2)=\left[\begin{array}{ll}
0.1 & -0.1
\end{array}\right], M_{2}(3)=\left[\begin{array}{ll}
0 & -0.1
\end{array}\right], M_{3}(1)=\left[\begin{array}{cc}
-0.1 & 0.1
\end{array}\right], M_{3}(2)=\left[\begin{array}{ll}
0.2 & -0.1
\end{array}\right], \\
& M_{3}(3)=\left[\begin{array}{ll}
0.1 & 0.1
\end{array}\right] ; M_{4}(1)=\left[\begin{array}{ll}
-0.1 & 0.1
\end{array}\right], M_{4}(2)=\left[\begin{array}{ll}
0.1 & -0.2
\end{array}\right], M_{4}(3)=\left[\begin{array}{ll}
0.2 & 0
\end{array}\right] .
\end{aligned}
$$

With the above parameters, by resorting to the Matlab toolbox, the matrix inequalities in (30)-(31) are solvable with feasible solution as follows (here only some of the matrices are listed for space saving):

$$
\begin{aligned}
& \varepsilon_{1}=13.3632, \quad \varepsilon_{2}=50.4805, \quad \delta_{1}=14.4284, \quad \delta_{2}=13.4711, \\
& P_{1}(1)=\left[\begin{array}{cc}
14.5824 & 1.7032 \\
1.7032 & 15.5435
\end{array}\right], P_{2}(2)=\left[\begin{array}{cc}
14.4964 & 0.8935 \\
0.8935 & 14.8581
\end{array}\right], P_{3}(3)=\left[\begin{array}{cc}
14.8398 & 1.4817 \\
1.4817 & 14.8786
\end{array}\right], \\
& P_{4}(1)=\left[\begin{array}{cc}
14.5694 & 1.5955 \\
1.5955 & 14.9914
\end{array}\right], P_{5}(2)=\left[\begin{array}{cc}
14.4554 & 0.9990 \\
0.9990 & 14.8456
\end{array}\right], P_{6}(3)=\left[\begin{array}{cc}
14.8805 & 1.4923 \\
1.4923 & 14.8647
\end{array}\right], \\
& P_{7}(1)=\left[\begin{array}{cc}
14.5396 & 1.6435 \\
1.6435 & 15.0343
\end{array}\right], Q_{11}=\left[\begin{array}{cc}
8.9461 & -0.0047 \\
-0.0047 & 8.9307
\end{array}\right] .
\end{aligned}
$$

From Theorem 2, we know that system (6) is a robustly distributed state estimator of the Markov-type dynamic system (1) with measurement outputs (2). The estimation gain matrices can be obtained from (32) as follows

$$
\begin{aligned}
& K_{11}(1)=\left[\begin{array}{cc}
1.1380 & 0.5299 \\
-0.1974 & -0.8014
\end{array}\right], K_{12}(1)=\left[\begin{array}{ll}
0.0211 & 0.0869 \\
0.0213 & 0.0877
\end{array}\right], K_{13}(1)=\left[\begin{array}{cc}
0.0407 & -0.0113 \\
0.0402 & -0.0111
\end{array}\right], \\
& K_{14}(1)=\left[\begin{array}{ll}
0.0648 & -0.0235 \\
0.0690 & -0.0250
\end{array}\right], K_{22}(1)=\left[\begin{array}{cc}
-1.3023 & -0.2051 \\
0.6984 & -0.2023
\end{array}\right], K_{33}(1)=\left[\begin{array}{cc}
1.4388 & 0.1756 \\
-0.8127 & -0.3919
\end{array}\right], \\
& K_{44}(1)=\left[\begin{array}{cc}
-1.2707 & 1.5833 \\
0.5028 & -1.0010
\end{array}\right], K_{53}(1)=\left[\begin{array}{cc}
0.0532 & -0.0147 \\
-0.0883 & 0.0244
\end{array}\right], K_{54}(1)=\left[\begin{array}{cc}
0.0853 & -0.0309 \\
-0.1433 & 0.0520
\end{array}\right], \\
& K_{55}(1)=\left[\begin{array}{cc}
2.3527 & -1.5978 \\
-1.2534 & 1.3268
\end{array}\right], K_{56}(1)=\left[\begin{array}{cc}
0.2769 & 0.2854 \\
-0.4593 & -0.4735
\end{array}\right], K_{66}(1)=\left[\begin{array}{cc}
1.1286 & 2.6005 \\
-1.1294 & -1.6011
\end{array}\right] ;
\end{aligned}
$$




$$
\begin{aligned}
& K_{11}(2)=\left[\begin{array}{cc}
0.9209 & -1.0391 \\
-1.8212 & -0.9115
\end{array}\right], K_{12}(2)=\left[\begin{array}{cc}
-0.1467 & 0.3593 \\
0.2878 & -0.7048
\end{array}\right], K_{13}(2)=\left[\begin{array}{cc}
-0.0176 & -0.1056 \\
0.0371 & 0.2207
\end{array}\right], \\
& K_{14}(2)=\left[\begin{array}{cc}
-0.0714 & -0.0329 \\
0.1442 & 0.0663
\end{array}\right], K_{22}(2)=\left[\begin{array}{cc}
0.2918 & 0.8373 \\
-0.4807 & -0.2707
\end{array}\right], K_{33}(2)=\left[\begin{array}{cc}
-0.6959 & 0.3358 \\
-0.3032 & -0.3285
\end{array}\right], \\
& K_{44}(2)=\left[\begin{array}{cc}
-0.7166 & 0.6706 \\
-0.5977 & -0.0851
\end{array}\right], K_{53}(2)=\left[\begin{array}{cc}
-0.0009 & -0.0051 \\
-0.0021 & -0.0113
\end{array}\right], K_{54}(2)=\left[\begin{array}{cc}
-0.0047 & -0.0021 \\
-0.0104 & -0.0046
\end{array}\right], \\
& K_{55}(2)=\left[\begin{array}{cc}
1.0006 & 0 \\
-0.2424 & 0
\end{array}\right], K_{56}(2)=\left[\begin{array}{cc}
-0.3429 & 0.6859 \\
0.0602 & -0.1203
\end{array}\right], K_{66}(2)=\left[\begin{array}{cc}
-1.0012 & 0.5051 \\
-0.0103 & 0.0150
\end{array}\right]
\end{aligned}
$$

and

$$
\begin{aligned}
& K_{11}(3)=\left[\begin{array}{cc}
-0.4937 & 0.8411 \\
0.4937 & -0.8392
\end{array}\right], K_{12}(3)=\left[\begin{array}{cc}
0.0387 & -0.0080 \\
-0.0381 & 0.0079
\end{array}\right], K_{13}(3)=\left[\begin{array}{cc}
-0.7443 & -0.3208 \\
0.7445 & 0.3208
\end{array}\right], \\
& K_{14}(3)=\left[\begin{array}{cc}
-0.1473 & -0.0732 \\
0.1491 & 0.0741
\end{array}\right], K_{22}(3)=\left[\begin{array}{cc}
-0.7439 & -0.0522 \\
0.7429 & 0.0524
\end{array}\right], K_{33}(3)=\left[\begin{array}{cc}
0.4997 & -0.0776 \\
-0.4970 & 0.0787
\end{array}\right], \\
& K_{44}(3)=\left[\begin{array}{cc}
-0.0001 & -1.0000 \\
0.0040 & 1.0020
\end{array}\right], K_{53}(3)=\left[\begin{array}{cc}
0.0001 & 0.0001 \\
-0.0112 & -0.0049
\end{array}\right], K_{54}(3)=\left[\begin{array}{cc}
0.0000 & 0.0000 \\
-0.0005 & -0.0003
\end{array}\right], \\
& K_{55}(3)=\left[\begin{array}{cc}
-0.2948 & -0.5892 \\
0.3097 & 0.5892
\end{array}\right], K_{56}(3)=\left[\begin{array}{cc}
0.0000 & 0.0000 \\
-0.0009 & -0.0011
\end{array}\right], K_{66}(3)=\left[\begin{array}{cc}
0.2764 & 0.4779 \\
-0.2757 & -0.4770
\end{array}\right] .
\end{aligned}
$$

Remark 3: In this paper, the sensor network consists of $n$ modes and the network switches from one mode to another according to a Markov chain with known transition probability. In our future work, we could further consider two independent Markovian jumping modes for node switching as well as network topology switching, which would reflect more features of the complexity. On the other hand, the topology for many sensor networks in a real world can be described by a random graph and the study about random dynamical networks is therefore quite interesting and useful. One of our future research topics would be the extension of our results to random dynamical networks.

\section{Conclusions}

In this paper, we have studied the state estimation problem for a class of Markov-type uncertain dynamical systems with mode-dependent distributed delays. Through the measurement outputs obtained by the sensors in sensor networks, a distributed state estimator has been designed to estimate the states of the dynamical plant. By resorting to the Lyapuonv stability theory and the matrix inequality techniques, sufficient criteria have been obtained to ensure the estimation error system to be robustly asymptotically stable in the mean square sense which in turn show that the designed estimator is a robustly distributed state estimator of the original plant system. Furthermore, the estimation gains have been explicitly characterized by the solution of the matrix inequality conditions. Finally, an illustrative example has been given to demonstrate the

effectiveness of our results. Future research topics include the extension of the present results to other systems such as fuzzy systems [30,31] and networked control systems [17, 17, 23, 25].

\section{REFERENCES}

[1] E. K. Boukas and Z. K. Liu, Robust $H_{\infty}$ control of discrete-time Markovian jump linear systems with mode-dependent time-delays, IEEE Trans. Automat. Control, Vol. 46, pp. 1918-1924, 2001. 
[2] S. Boyd, L. E. Ghaoui, E. Feron and V. Balakrishnan, Linear Matrix Inequalities in System and Control Theory, Philadelphia: SIAM, 1994.

[3] R. Brooks, P. Ramanathan and A. M. Sayeed, Distributed target classification and tracking in sensor networks, Proceedings of the IEEE, Vol. 91, No. 8, pp. 1163-1171, 2003.

[4] C. Busch, M. Magdon-Ismail and M. Mavronicolas, Efficient bufferless packet switching on trees and leveled networks, Journal of Parallel and Distributed Computing, Vol. 67, No. 11, pp. 1168-1186, 2007.

[5] F. S. Cattivelli and A. H. Sayed, Diffusion LMS strategies for distributed estimation, IEEE Trans. Signal Processing, Vol. 58, No. 3, pp. 1035-1048, 2010.

[6] F. S. Cattivelli and A. H. Sayed, Diffusion strategies for distributed Kalman filtering and smoothing, IEEE Trans. Automatic Control, DOI 10.1109/TAC.2010.2042987, to appear, 2010.

[7] L. Chai, B. Hu and P. Jiang, Distributed state estimation based on quantized observations in a bandwidth constrained sensor network, Proc. 7th World Congress on Intelligent Control and Automation, pp. 2411-2415, 2008.

[8] W.-M. Chen, C.-S. Li, F.-Y. Chiang and H.-C. Chao, Jumping ant routing algorithm for sensor networks, Computer Communications, Vol. 30, pp. 2892-2903, 2007.

[9] H. Gao and T. Chen, $H_{\infty}$ estimation for uncertain systems with limited communication capacity, IEEE Trans. Automat. Control, Vol. 52, No. 11, pp. 2070-2084, 2007.

[10] I. Gunawan, Reliability analysis of shuffle-exchange network systems, Reliability Engineering and System Safety, Vol. 93, pp. 271-276, 2008.

[11] J. H. Kim, M. West, E. Scholte and S. Narayanan, Multiscale consensus for decentralized estimation and its application to building systems, Proc. American Control Conference, pp. 888-893, 2008.

[12] H. Liu, F. Sun, K. He and Z. Sun, Design of reduced-order $\mathcal{H}_{\infty}$ filter for markovian jumping systems with time delay, IEEE Trans. Circuits and Systems - II, Vol. 51, No. 11, pp. 1837-1841, 2006.

[13] Y. Liu, Z. Wang and X. Liu, State estimation for discrete-time Markovian jumping neural networks with mixed modedependent delays, Phys. Lett. A, Vol. 372, pp. 7147-7155, 2008.

[14] Y. Liu, Z. Wang and X. Liu, Exponential synchronization of complex networks with Markovian jump and mixed delays, Phys. Lett. A, Vol. 372,pp. 3986-3998, 2008.

[15] Y. Liu, Z. Wang, J. Liang and X. Liu, Stability and synchronization of discrete-time Markovian jumping neural networks with mixed mode-dependent time delays, IEEE Trans. Neural Networks, Vol. 20, No. 7, pp. 1102-1116, 2009.

[16] Y. Liu, Z. Wang, J. Liang and X. Liu, Synchronization and state estimation for discrete-time complex networks with distributed delays, IEEE Trans. Syst. Man Cybern., Vol. 38, No. 5, pp. 1314-1325, 2008.

[17] D. Martin, R. del Toro, R. Haber and J. Dorronsoro, Optimal tuning of a networked linear controller using a multi-objective genetic algorithm and its application to one complex electromechanical process, International Journal of Innovative Computing, Information and Control, Vol. 5, No. 10(B), pp. 3405-3414, 2009.

[18] R. Olfati-Saber, Distributed Kalman filtering for sensor networks, Proc. 46th IEEE Conf. Decision and Control, New Orleans, LA, 2007.

[19] R. Olfati-Saber and J. S. Shamma, Consensus filters for sensor networks and distributed sensor fusion, Proc. 44th IEEE Conf. Decision and Control, and the Euro. Cont. Conf., Seville, Spain, 2005.

[20] M. Patan and D. Ucinski, Conguration of sensor network with uncertain location of nodes for parameter estimation in distributed parameter systems, Proc. 14th International Conference on Methods and Models in Automation and Robotics, Vol. 14, No. 1, 2009.

[21] P. Shi, M. Mahmoud, S. K. Nguang and A. Ismail, Robust filtering for jumping systems with mode-dependent delays, Signal Processing, Vol. 86, No. 1, pp. 140-152, 2006.

[22] A. Speranzon, C. Fischione, K. H. Johansson and A. Sangiovanni-Vincentelli, A distributed minimum variance estimator for sensor networks, IEEE Journal on Selected Areas in Communications, Vol. 26, No. 4, pp. 609-621, 2008.

[23] V. Vesely and T. N. Quang, Robust output networked control system design, ICIC Express Letters, Vol. 4, No. 4, pp. 1399$1407,2010$.

[24] L. Wu, P. Shi, H. Gao and C. Wang, $\mathcal{H}_{\infty}$ filtering for 2D Markovian jump systems, Automatica, Vol. 44, No. 7, pp. 1849-1858, 2008.

[25] Y. Xia, Z. Zhu and M. S. Mahmoud, $H_{2}$ control for networked control systems with Markovian data losses and delays, ICIC Express Letters, Vol. 3, No. 3(A), pp. 271-276, 2009.

[26] J. Xiong and J. Lam, Fixed-order robust $\mathcal{H}_{\infty}$ filter design for Markovian jump systems with uncertain switching probabilities, IEEE Trans. Signal Processing, Vol. 54, No. 4, pp. 1421-1430, 2006. 
[27] Z. Xiong, A. D. Liveris and S. Cheng, Distributed source coding for sensor networks, IEEE Signal Processing Magazine, pp. 80-94, 2004.

[28] W. Yu, G. Chen, Z. Wang, and W. Yang, Distributed consensus filtering in sensor networks, IEEE Trans. Syst. Man \& Cybern.-B, Vol. 39, No. 6, pp. 1568-1577, 2009.

[29] H. Zhang, G. Feng, G. Duan and X. Lu, $\mathcal{H}_{\infty}$ filtering for multiple-time-delay measurements, IEEE Trans. Signal Processing, Vol. 54, No. 5, pp. 1681-1688, 2006.

[30] Y. Zhao, H. Gao, J. Lam and B. Du, Stability and atabilization of delayed T-S fuzzy systems: a delay partitioning approach, IEEE Transactions on Fuzzy Systems, Vol. 17, No. 4, pp. 750-762, 2009.

[31] Y. Zhao, J. Wu and P. Shi, $H_{\infty}$ control of nonlinear dynamic systems: a new fuzzy delay fractioning approach, IET Control Theory \& Applications, Vol. 3, No. 7, pp. 917-928, 2009.

[32] X. Zhu, C. Hua and S. Wang, State feedback controller design of networked control systems with time delay in the plant, International Journal of Innovative Computing, Information and Control, Vol. 4, No. 2, pp. 283-290, 2008. 